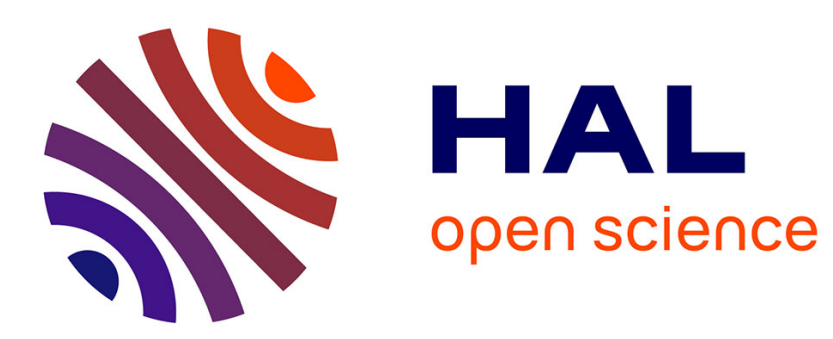

\title{
Distribution of the span of 1-dimensional confined random processes before hitting a target
}

\author{
J. Klinger, R. Voituriez, O. Bénichou
}

\section{To cite this version:}

J. Klinger, R. Voituriez, O. Bénichou. Distribution of the span of 1-dimensional confined random processes before hitting a target. Physical Review E , 2021, 103 (3), 10.1103/PhysRevE.103.032107. hal-03436729

\section{HAL Id: hal-03436729 \\ https://hal.science/hal-03436729}

Submitted on 19 Nov 2021

HAL is a multi-disciplinary open access archive for the deposit and dissemination of scientific research documents, whether they are published or not. The documents may come from teaching and research institutions in France or abroad, or from public or private research centers.
L'archive ouverte pluridisciplinaire HAL, est destinée au dépôt et à la diffusion de documents scientifiques de niveau recherche, publiés ou non, émanant des établissements d'enseignement et de recherche français ou étrangers, des laboratoires publics ou privés. 


\title{
Distribution of the span of 1-dimensional confined random processes before hitting a target
}

\author{
J. Klinger and R. Voituriez \\ Laboratoire de Physique Théorique de la Matière Condensée, \\ UMR 7600 CNRS /UPMC, 4 Place Jussieu, 75255 Paris Cedex, France and \\ Laboratoire Jean Perrin, UMR 8237 CNRS /UPMC, 4 Place Jussieu, 75255 Paris Cedex \\ O. Bénichou \\ Laboratoire de Physique Théorique de la Matière Condensée, \\ UMR 7600 CNRS /UPMC, \& Place Jussieu, 75255 Paris Cedex, France
}

\begin{abstract}
We derive the distribution of the number of distinct sites visited by a random walker before hitting a target site of a finite $1 d$ domain. Our approach holds for the general class of Markovian processes with connected span - ie whose trajectories have no "holes". We show that the distribution can be simply expressed in terms of splitting probabilities only. We provide explicit results for classical examples of random processes with relevance to target search problems, such as simple symmetric random walks, biased random walks, persistent random walks and resetting random walks. As a byproduct, explicit expressions for the splitting probabilities of all these processes are given. Extensions to reflecting boundary conditions, continuous processes, and an example of random process with non connected span are discussed.
\end{abstract}

\section{INTRODUCTION}

Quantifying the exploration of a domain by a random walker is a long standing question, which has applications in a variety of problems, ranging from living organisms exploring their environment to find ressources, to robots designed to accomplish specific tasks such as cleaning or demining [1-3]. In this context, the determination of first-passage time statistics to target sites of interest in a domain has played an important role in the literature [4-7]. The case of confined geometries has proved to be particularly relevant in the field of target search problems, and has been the focus of many theoretical works [8-13]. Beyond first-passage times, other observables, such as cover times - time needed to explore exhaustively a domain of interest [14-16]- or occupation times - cumulative time spent in a sub domain of interest - have also been studied [17-21].

Another classical quantifier of random exploration is given, in a discrete setting, by the number of distinct sites visited by a random walker after $n$ steps $[1,22,23]$. In this paper, we focus on a related observable $S$ that is defined as the number of distinct sites visited by a random walker before hitting a target site of a finite domain, where it is assumed to be either trapped or removed. The observable $S$ therefore quantifies the fraction of the domain that has been explored before a target is found, which provides a further characterization of random search processes. Note that this observable does not seem to have received much attention in the literature, with the notable exception of [24], where it was studied for a continuous one dimensional unconfined system.

Here we focus on 1-dimensional geometries with periodic boundary conditions, and derive the full distribution of $S$ for the general class of Markovian processes with connected span - i.e whose trajectories have no "holes". We show that the explicit determination of this distribution amounts to calculating splitting probabilities [4], i.e probabilities that the walker reaches a given site $x_{1}$ before a second site $x_{2}$. We next apply these results to classical examples of random processes with relevance to target search problems, such as simple symmetric random walks, biased random walks, persistent random walks and resetting random walks $[18,25,26]$. As a by-product of our study, we provide explicit expressions for the splitting probabilities of all these processes, some of which were not known to the best of our knowledge. Finally, we extend our results to the case of reflecting boundary conditions, continuous processes, and an example of random process with non connected span.

\section{GENERAL EXPRESSION OF THE DISTRIBUTION OF $S$}

In what follows, unless specified otherwise, we consider a Markovian random walker on a $1 d$ periodic lattice of $N$ sites, labelled from 0 to $N-1$. We denote by 0 the target (or exit) site, and by $s_{0}$ the starting position. Let $S$ be the number of distinct sites visited by a trajectory that ends at 0 , where by convention 0 and $s_{0}$ are included; see Fig. 1.

Importantly, we consider only processes with connected span, for which unvisited sites can only be reached by nearest neighbor jumps, so that qualitatively trajectories have no holes.

We first consider the case of a semi infinite lattice (equivalent to the limit $N \rightarrow \infty$ ). The probability for the walker to have visited exactly $n$ sites when it escapes the domain is then exactly given by the probability that it reaches the site $n-1$, and then escapes without going 


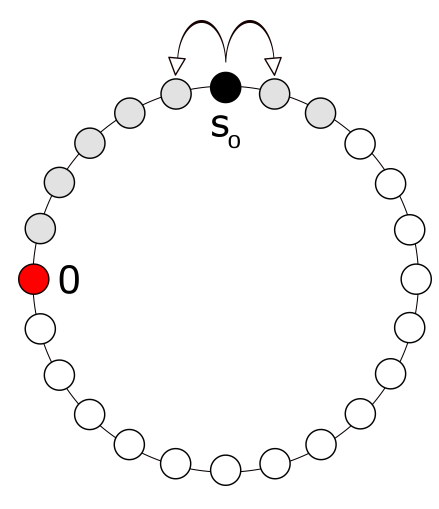

Figure 1. Number of distinct sites visited by a random walker before hitting a target site of a finite domain. Starting from $s_{0}$, the random walker visits all the light gray sites before reaching the red target site 0 . In this case, $N=24$ and $S=9$.

any further than $n-1$; see Fig. 2. In other words, one

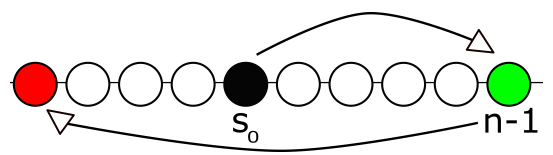

Figure 2. Example of trajectory. Starting from $s_{0}$, the walker first reaches site $n-1$ then goes back on its tracks to exit at the red site.

has:

$$
P(S=n)=\pi_{\underline{n-1}, 0}\left(s_{0}\right) \quad \pi_{\underline{0}, n}(n-1)
$$

where the Markov property has been used. Here $\pi_{\underline{i}, j}(k)$ is the splitting probability that the walker, starting from $k$, reaches the site $i$ before the site $j$. The exact distribution of $S$ for the walker in a finite $1 d$ periodic geometry is obtained by using the same idea. Taking into account the fact that $S$ is bounded by $N$ and that the trajectories to the target can be sorted as either "clockwise" or "counter-clockwise", we finally obtain:

$$
\begin{aligned}
P(S=n)= & 1_{s_{0}+1 \leq n} \pi_{\underline{n-1,0},}\left(s_{0}\right) \pi_{\underline{0}, n}(n-1)+ \\
& 1_{n \geq N-s_{0}+1} \pi_{\underline{N-n+1}, N}\left(s_{0}\right) \pi_{\underline{N}, N-n}(N-n+1)
\end{aligned}
$$

where $1_{s_{0}+1 \leq n}=1$ if $s_{0}+1 \leq n$ and $1_{s_{0}+1 \leq n}=0$ otherwise; $1_{n \geq N-s_{0}+1}$ is defined accordingly. Importantly, the expression (2) is exact for all Markov processes with connected span and can be easily extended to one step non-Markovian processes, such as the persistent walk considered below. In addition it is fully explicit, provided that the splitting probabilities of the process can be determined. Below, we now provide explicit examples.

\section{APPLICATIONS : EXPLICIT EXAMPLES}

\section{A. Symmetric nearest neighbor random walk}

Let us first consider the example of the classical symmetric nearest neighbor random walk. As mentioned above, this case has been studied in the limit $N \rightarrow \infty$ (unconfined system) in the continuous space approximation in [24]. The splitting probability is well known $[1,27]$, and given by:

$$
\begin{aligned}
& 0 \leq s_{0} \leq s_{1}, \pi_{\underline{s_{1}}, 0}\left(s_{0}\right)=\frac{s_{0}}{s_{1}} \\
& s_{1} \leq s_{0} \leq N, \pi_{\underline{s_{1}}, N}\left(s_{0}\right)=\frac{N-s_{0}}{N-s_{1}}
\end{aligned}
$$

The distribution of $S$ is then explicitly determined by (2). Figure 3 shows the exact distribution $P(S)$ (confirmed by numerical simulations) for examples of parameters $s_{0}$ and $N$. Note the two sharp jumps in the distribution located at $n=s_{0}+1$ and $n=N-s_{0}+1$, which correspond to the minimal number of sites visited by the two classes of clockwise and counterclockwise trajectories. As expected, these sharp jumps are observed for $s_{0}=O(N)$ and $N-s_{0}=O(N)$, and the distribution smoothens for $N \gg s_{0}$ (or equivalently $N \gg N-s_{0}$ ), where the semi infinite case is recovered.
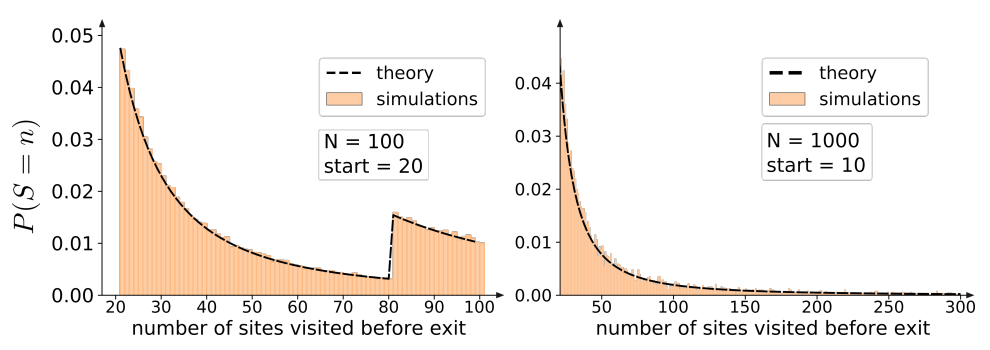

Figure 3. Distribution $P(S)$ for a symmetric nearest neighbor random walker. Left: $s_{0}=20, N=100$ - Right: $s_{0}=10, N=$ 1000. Exact results are compared to $10^{6}$ numerical simulations on the left and $10^{5}$ on the right.

\section{B. Biased nearest neighbor random walk}

In many physical situations, external force fields can give rise to biased diffusion. The corresponding model is that of the biased random walk, defined in a discrete setting as follows: at each step the walker steps to the right with probability $p$ and to the left with probability $1-p$ [1]. The splitting probability can be obtained from the classical backward equation $[1,4]$, which yields:

$$
\begin{aligned}
& 0 \leq s_{0} \leq s_{1}, \pi_{\underline{s_{1}}, 0}\left(s_{0}\right)=\frac{\alpha^{s_{0}}-1}{\alpha^{s_{1}}-1} \\
& s_{1} \leq s_{0} \leq N, \pi_{\underline{s_{1}}, N}\left(s_{0}\right)=\frac{\alpha^{s_{0}}-\alpha^{N}}{\alpha^{s_{1}}-\alpha^{N}}
\end{aligned}
$$


where $\alpha=\frac{1-p}{p}$. The distribution of $S$ is then explicitly determined by (2). Similarly to the unbiased case above, the distribution shows two sharp jumps for $s_{0}=O(N)$ and $N-s_{0}=O(N)$, whose interpretation is unchanged; their relative weight is now controlled by the bias (see Fig 4).

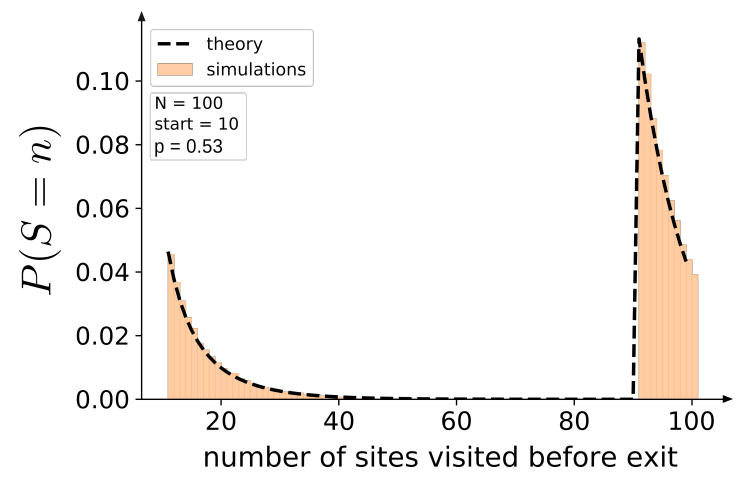

Figure 4. Distribution $P(S)$ for a biased random walker starting at $s_{0}=10$ with rightward drift $p=0.53$. The rightmost peak has much higher weight because the walker has a strong tendency to escape clockwise, even though the counterclockwise exit is closer. Exact results are compared to $10^{5}$ numerical simulations.

\section{Persistent nearest neighbor random walk}

Another important example of random walk involved in the context of search processes is the persistent random walk $[22,28]$, defined as follows. At each time step the walker performs a step identical to the previous one with probability $p$, and opposite with probability $1-p$. We also introduce the probability $a$ that the first step is clockwise. Even though its derivation relies on rather standard tools, the expression of the splitting probability for the persistent random walk does not seem to be present in the literature; we therefore provide the main steps of the derivation below. We consider the case

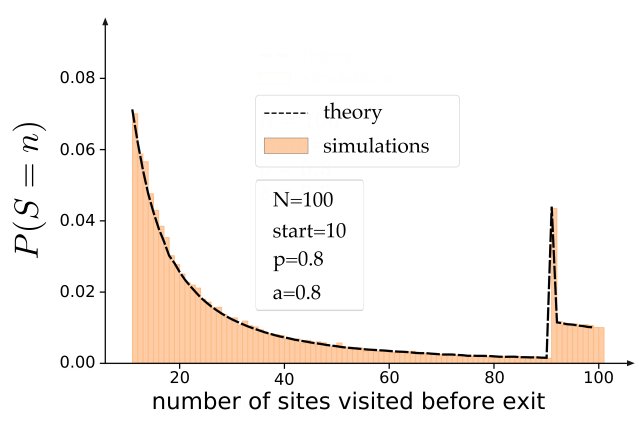

Figure 5. Distribution $P(S)$ for a persistent random walker with $s_{0}=10, N=100, p=0.8$ and $a=0.8$. Exact results are compared to $5.10^{5}$ numerical simulations. $0<s_{0}<s$ and aim at determining $\pi_{\underline{s}, 0}\left(s_{0}, a\right)$; the case $s<s_{0}<N$ will then be deduced by taking $s \rightarrow N-s$, $s_{0} \rightarrow N-s_{0}$ and $a \rightarrow 1-a$. Boundary conditions yield $\pi_{\underline{s}, 0}(s, a)=1$ and $\pi_{\underline{s}, 0}(0, a)=0$. It is useful to define $u_{\underline{s}, 0}\left(s_{0}\right)$ as the probability to reach $s$ before 0 knowing that the step that led to $s_{0}$ was to the right, with the boundary condition $u_{\underline{s}, 0}(s)=1$. Similarly, we introduce $v_{\underline{s}, 0}\left(s_{0}\right)$ as the probability to reach $s$ before 0 knowing that the step that led to $s_{0}$ was to the left, with the boundary condition $v_{\underline{s}, 0}(0)=0$. Partitioning over the first step of the walk, we obtain the following equation satisfied by $\pi_{\underline{s}, 0}\left(s_{0}, a\right)$ for $0<s_{0}<s$ :

$$
\pi_{\underline{s}, 0}\left(s_{0}, a\right)=a u_{\underline{s}, 0}\left(s_{0}+1\right)+(1-a) v_{\underline{s}, 0}\left(s_{0}-1\right)
$$

Similarly, a set of equations for $u$ and $v$ is given by:

$$
\begin{aligned}
& u_{\underline{s}, 0}\left(s_{0}\right)=p u_{\underline{s}, 0}\left(s_{0}+1\right)+(1-p) v_{\underline{s}, 0}\left(s_{0}-1\right) \\
& v_{\underline{s}, 0}\left(s_{0}\right)=p v_{\underline{s}, 0}\left(s_{0}-1\right)+(1-p) u_{\underline{s}, 0}\left(s_{0}+1\right)
\end{aligned}
$$

which can be rewritten as:

$$
\begin{aligned}
& u_{\underline{s}, 0}\left(s_{0}+1\right)-2 u_{\underline{s}, 0}\left(s_{0}\right)+u_{\underline{s}, 0}\left(s_{0}-1\right)=0 \\
& v_{\underline{s}, 0}\left(s_{0}\right)=\frac{1}{1-p}\left(u_{\underline{s}, 0}\left(s_{0}+1\right)-p u_{\underline{s}, 0}\left(s_{0}+2\right)\right)
\end{aligned}
$$

Enforcing the boundary conditions leads to the following determination of $u_{\underline{s}, 0}\left(s_{0}\right), v_{\underline{s}, 0}\left(s_{0}\right)$ :

$$
\begin{aligned}
& u_{\underline{s}, 0}\left(s_{0}\right)=1+B\left(s_{0}-s\right) \\
& v_{\underline{s}, 0}\left(s_{0}\right)=B s_{0}
\end{aligned}
$$

where

$$
B=\frac{p-1}{(1-s)(1-p)-p}
$$

Finally, the splitting probability reads for $0<s_{0}<s$ :

$$
\pi_{\underline{s}, 0}\left(s_{0}, a\right)=B s_{0}+a(1+2 B-B s)-B
$$

or equivalently

$$
\pi_{\underline{s}, 0}\left(s_{0}, a\right)=\frac{p-1}{(1-p)(1-s)-p} s_{0}+\frac{1-p-a}{1+p(s-2)-s}
$$

Note that the splitting probability is an affine function of $s_{0}$, as in the case of the normal random walk. However, the slope for the persistent random walk is controlled by both $p$ and $s$. As expected, the case of the normal walk is recovered by taking $p=a=1 / 2$. The distribution of $S$ is then explicitly determined by (2) and reads:

$$
\begin{aligned}
& P(S=n)= \\
& 1_{s_{0}+1 \leq n} \pi_{n-1,0}\left(s_{0}, a\right) \pi_{\underline{0}, n}(n-1, p)+ \\
& 1_{n \geq N-s_{0}+1} \underline{\underline{N-n+1, N}}\left(s_{0}, a\right) \pi_{\underline{N}, N-n}(N-n+1,1-p)
\end{aligned}
$$

Figure 5 shows $P(S)$ for $a=0.8$ and $p=0.8$. Again, the distribution shows two sharp jumps for $s_{0}=O(N)$ and $N-s_{0}=O(N)$. Here, the corresponding peaks are sharpened as the persistence time of the random walk (controlled by the parameter $p$ ) is increased. 


\section{Resetting random walk}

We now turn to the resetting walk, which is another example of search process that has recently been given much attention $[25,26]$. This process has been mostly studied in continuous space and time; we here introduce a discrete version. At each time step, the walker either performs a nearest neighbor jump (drawn symmetrically) with probability $1-\lambda$, or resets to its initial position $s_{0}$ with probability $\lambda$. Note that despite the resetting jumps whose range can cover many sites, the span of the process remains connected, because unvisited sites can only be reached by nearest neighbor jumps (note that resetting jumps all lead to the initial site).

We first determine the splitting probability $\pi_{\underline{s}, 0}\left(s_{0}\right)$, which has not been given explicitly in the literature for discrete processes to the best of our knowledge, even if related quantities have been studied for continuous resetting processes $[29,30]$. It is convenient to introduce an auxiliary site $s_{p}$ (not necessarily equal to $s_{0}$ ), where resetting jumps end. The Backward equation satisfied by $\pi_{\underline{s}, 0}\left(s_{0}\right)$ can then be written:

$\pi_{\underline{s}, 0}\left(s_{0}\right)=\frac{1-\lambda}{2}\left(\pi_{\underline{s}, 0}\left(s_{0}+1\right)+\pi_{\underline{s}, 0}\left(s_{0}-1\right)\right)+\lambda \pi_{\underline{s}, 0}\left(s_{p}\right)$

or equivalently

$$
L_{s_{0}} \pi_{\underline{s}, 0}\left(s_{0}\right)=-\frac{2 \lambda}{1-\lambda} \pi_{\underline{s}, 0}\left(s_{p}\right)
$$

with

$$
L_{s_{0}} \pi_{\underline{s}, 0}\left(s_{0}\right)=\pi_{\underline{s}, 0}\left(s_{0}+1\right)-\frac{2}{1-\lambda} \pi_{\underline{s}, 0}\left(s_{0}\right)+\pi_{\underline{s}, 0}\left(s_{0}-1\right)
$$

The Backward equation is completed by the boundary conditions $\pi_{\underline{s}, 0}(0)=0, \pi_{\underline{s}, 0}(s)=1$. The solution of the linear equation (16) is then given by the sum of the homogeneous solution $h_{s}\left(s_{0}\right)$ and a particular solution $p\left(s_{0}\right)$. The homogeneous solution, defined by

$$
L_{s_{0}} h_{s}\left(s_{0}\right)=0
$$

and $h_{s}(0)=0, h_{s}(s)=1$ can be written

$$
h_{s}\left(s_{0}\right)=\frac{r_{+}^{s_{0}}-r_{-}^{s_{0}}}{r_{+}^{s}-r_{-}^{s}}
$$

where $r_{ \pm}=\frac{1}{1-\lambda} \pm \sqrt{\frac{1}{(1-\lambda)^{2}}-1}$. In turn, the particular solution can be constructed using the green function of the problem defined by:

$$
L_{s_{1}} G\left(s_{1}, s_{2}\right)=\delta_{s_{1}, s_{2}}
$$

with vanishing boundary conditions

$$
G\left(0, s_{2}\right)=G\left(s, s_{2}\right)=0
$$

One obtains

$$
G\left(s_{1}, s_{2}\right)=1_{s_{1} \leq s_{2}} G_{-}\left(s_{1}, s_{2}\right)+1_{s_{1}>s_{2}} G_{+}\left(s_{1}, s_{2}\right)
$$

where

$$
\begin{aligned}
& G_{-}\left(s_{1}, s_{2}\right)=A\left(s_{2}\right)^{-1}\left(r_{+}^{s_{1}}-r_{-}^{s_{1}}\right) \\
& G_{+}\left(s_{1}, s_{2}\right)=A\left(s_{2}\right)^{-1} \frac{r_{+}^{s_{2}}-r_{-}^{s_{2}}}{r_{+}^{s_{2}}-r_{-}^{s_{2}} \frac{r_{+}^{s}}{r_{-}^{s}}}\left(r_{+}^{s_{1}}-r_{-}^{s_{1}} \frac{r_{+}^{s}}{r_{-}^{s}}\right)
\end{aligned}
$$

and

$$
\begin{aligned}
A\left(s_{2}\right)= & \left(r_{+}^{s_{2}}-r_{-}^{s_{2}}\right)\left(r_{+}^{s_{2}}-r_{-}^{s_{2}} \frac{r_{+}^{s}}{r_{-}^{s}}\right)^{-1}\left(r_{+}^{s_{2}+1}-r_{-}^{s_{2}+1} \frac{r_{+}^{s}}{r_{-}^{s}}\right) \\
& -\frac{2}{1-\lambda}\left(r_{+}^{s_{2}}-r_{-}^{s_{2}}\right)+\left(r_{+}^{s_{2}-1}-r_{-}^{s_{2}-1}\right)
\end{aligned}
$$

Finally, the particular solution can be written

$$
p\left(s_{0}\right)=-\frac{2}{1-\lambda} \pi_{\underline{s}, 0}\left(s_{p}\right) \sum_{s_{2}} G\left(s_{0}, s_{2}\right)
$$

Taking now $s_{0}=s_{p}$ and writing $\pi_{\underline{s}, 0}\left(s_{0}\right)=h_{s}\left(s_{0}\right)+p\left(s_{0}\right)$ provides a self-consistent equation for $\pi_{\underline{s}, 0}\left(s_{p}\right)$, which finally yields

$$
\pi_{\underline{s}, 0}\left(s_{p}\right)=\frac{h_{s}\left(s_{p}\right)}{1+\frac{2 \lambda}{1-\lambda} \sum_{s_{2}} G\left(s_{p}, s_{2}\right)}
$$

The splitting probability for arbitrary $s_{p}$ and $s_{0}$ can then be obtained as:

$$
\pi_{\underline{s}, 0}\left(s_{0}\right)=h_{s}\left(s_{0}\right)-\frac{h_{s}\left(s_{p}\right) 2 \lambda \sum_{s_{2}} G\left(s_{0}, s_{2}\right)}{1-\lambda+2 \lambda \sum_{s_{2}} G\left(s_{p}, s_{2}\right)}
$$

which covers in particular the case of resetting to the initial position $s_{p}=s_{0}$ that we consider in this paper. Interestingly, as $\lambda$ increases, the splitting probability takes a step-like shape (see figure 6), which has important consequences on the distribution of the covered territory $S$, obtained as before from equation (2). Figure 7 reveals

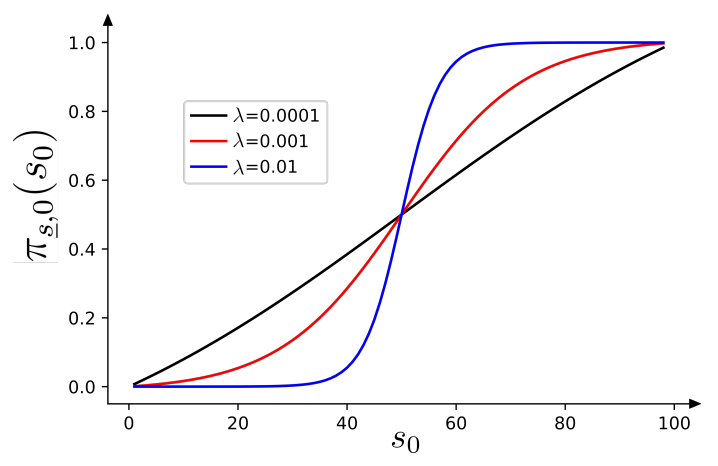

Figure 6. Splitting probability of a resetting random walker on the interval $[0,100]$, as a function of the initial position for different values of the resetting probability $\lambda$. Exact result of (25).

an interesting behavior: as $\lambda$ increases, a spike in the distribution grows at $2 s_{0}$. This can be interpreted as follows. Let us assume $s_{0}<N / 2$, and define $D$ as the 

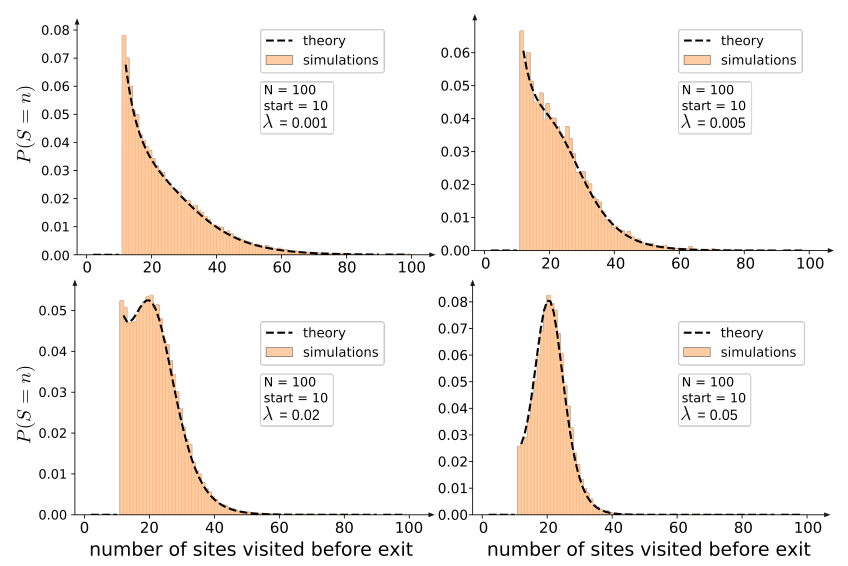

Figure 7. Distribution $P(S)$ for a resetting random walker, with $s_{0}=10$ and $N=100-(\mathrm{a}): \lambda=0.001,(\mathrm{~b}): \lambda=0.005$, (c): $\lambda=0.02,(d): \lambda=0.05$. Exact results are compared to $5.10^{5}$ numerical simulations.

discrete diffusion coefficient of the walk. As expected, for $\lambda \ll D / s_{0}^{2}$, resetting jumps can be neglected and one recovers the case of the symmetric nearest neighbor random walk. In turn, for $\lambda \gg D / s_{0}^{2}$, many resetting events, which lead to a symmetric exploration of the domain around $s_{0}$ occur before the target is reached. The explored territory before exit is thus approximately $2 s_{0}$, which yields the observed peak in the distribution.

\section{EXTENSIONS}

Let us summarize our results. So far, we have provided an analytical expression for the probability distribution of the territory explored before exit (equation (2)). This expression holds for discrete Markovian random processes with connected span on 1-dimensional periodic lattices. Below, we extend this result in several directions.

\section{A. Reflecting boundary condition}

We first consider the case of reflecting boundary conditions. We thus consider a discrete Markovian random process with connected span on a $1 d$ lattice of $N$ sites. The target site is still denoted by 0 , and we assume that there is a reflecting boundary at site $N-1$ : this effectively means that all nearest neighbor jumps from site $N-1$ lead to site $N-2$. The distribution of $S$ is then readily obtained and can be written:

$$
\begin{aligned}
& P(S=n)=0 \text { if } n \leq s_{0} \\
& P(S=n)=\pi_{\underline{n-1}, 0}\left(s_{0}\right) \pi_{\underline{0}, n}(n-1) \text { if } s_{0}<n<N \\
& P(S=n)=\pi_{\underline{N-1}, 0}\left(s_{0}\right) \text { if } n=N
\end{aligned}
$$

As in the case of periodic boundary conditions, this expression only involves splitting probabilities.

\section{B. Connection to the distribution of the maximum}

In this paragraph, we propose an alternative expression of the distribution of the territory explored before exit $P(S)$ for discrete random processes with connected span, in terms of the distribution $\sigma\left(s_{0}, s\right)$ (respectively $\left.\mu\left(s_{0}, s\right)\right)$ of the maximum (resp. minimum) $s$ reached by the random walker starting from $s_{0}$ positive (resp. negative) before exiting the domain at site 0 . In the case of periodic boundary conditions, it is clear that

$$
\begin{aligned}
P(S=n) & =1_{s_{0}+1 \leq n} \sigma\left(s_{0}, n-1\right) \\
& +1_{N-s_{0}+1 \leq n} \mu\left(s_{0}-N, 1-n\right)
\end{aligned}
$$

which takes the following simpler form in the case of a symmetric random walk:

$$
\begin{aligned}
P(S=n) & =1_{s_{0}+1 \leq n} \sigma\left(s_{0}, n-1\right) \\
& +1_{N-s_{0}+1 \leq n} \sigma\left(N-s_{0}, n-1\right)
\end{aligned}
$$

where $\sigma\left(s_{0}, s\right)$ can be simply expressed in terms of splitting probabilities according to

$$
\pi_{\underline{0}, s}\left(s_{0}\right)=\sum_{k=s_{0}}^{s-1} \sigma\left(s_{0}, k\right)
$$

Equivalently, one has

$$
\sigma\left(s_{0}, s\right)=\pi_{\underline{0}, s+1}\left(s_{0}\right)-\pi_{\underline{0}, s}\left(s_{0}\right)
$$

Note that the Markov property has not been used to obtain equation (28). For Markov processes with connected span, an alternative expression is given by:

$$
\sigma\left(s_{0}, s\right)=\pi_{\underline{s}, 0}\left(s_{0}\right) \pi_{\underline{0}, s+1}(s)
$$

which, together with equation (28) immediately yields equation (2). Note also that (31) and (30) yield the following equality valid for Markov processes:

$$
\pi_{\underline{0}, s+1}\left(s_{0}\right)-\pi_{\underline{0}, s}\left(s_{0}\right)=\pi_{\underline{s}, 0}\left(s_{0}\right) \pi_{\underline{0}, s+1}(s)
$$

which can be obtained by a direct probabilistic argument.

\section{Moments $\left\langle S^{p}\right\rangle$}

As a by-product, the expression (28) of the distribution $P(S)$ gives access to all moments of $\mathrm{S}$ according to:

$$
\begin{aligned}
\left\langle S^{p}\left(s_{0}\right)\right\rangle & =N^{p} \pi_{\underline{0}, N}\left(s_{0}\right)+N^{p} \pi_{\underline{0}, N}\left(N-s_{0}\right) \\
& -\sum_{n=s_{0}}^{N-1}\left((n+1)^{p}-n^{p}\right) \pi_{\underline{0}, n}\left(s_{0}\right) \\
& -\sum_{n=N-s_{0}}^{N-1}\left((n+1)^{p}-n^{p}\right) \pi_{\underline{0}, n}\left(N-s_{0}\right)
\end{aligned}
$$




\section{Continuous limit}

We now consider the continuous space limit of the problem; we are interested in continuous space random processes with connected span taking place on the $[0, L]$ ring with periodic boundary conditions. This can be readily done by making use of equation (27), which can be rewritten

$$
P(S=x)=1_{x_{0} \leq x} \sigma\left(x_{0}, x\right)+1_{L-x_{0} \leq x} \mu\left(x_{0}-L,-x\right)
$$

and in the symmetric case:

$$
P(S=x)=1_{x_{0} \leq x} \sigma\left(x_{0}, x\right)+1_{L-x_{0} \leq x} \sigma\left(L-x_{0}, x\right)
$$

where

$$
\sigma\left(x_{0}, x\right)=\frac{\mathrm{d}}{\mathrm{d} x} \pi_{\underline{0}, x}\left(x_{0}\right)
$$

Similarly to (33), any moment of $S$ can be written:

$$
\begin{aligned}
\left\langle S^{p}\left(x_{0}\right)\right\rangle & =\pi_{\underline{0}, L}\left(x_{0}\right) L^{p}+\pi_{\underline{0}, L}\left(L-x_{0}\right) L^{p} \\
& -p \int_{x_{0}}^{L} x^{p-1} \pi_{\underline{0}, x}\left(x_{0}\right) \mathrm{d} x \\
& -p \int_{L-x_{0}}^{L} x^{p-1} \pi_{\underline{0}, x}\left(L-x_{0}\right) \mathrm{d} x
\end{aligned}
$$

As an example, we consider the resetting random walk in continuous time and space with constant resetting rate $\lambda$ and diffusion constant $D$. The splitting probability obeys the following backward equation:

$$
\frac{\mathrm{d}^{2}}{\mathrm{~d} x^{2}} \pi_{\underline{x}, 0}\left(x_{0}\right)-r^{2} \pi_{\underline{x}, 0}\left(x_{0}\right)=r^{2} \pi_{\underline{x}, 0}\left(x_{p}\right)
$$

where $x_{p}$ denotes the resetting site, $x_{0}$ denotes the starting position, and $r=\sqrt{\frac{\lambda}{D}}$. The complete solution reads (see also [30]):

$$
\begin{aligned}
\pi_{\underline{x}, 0}\left(x_{0}\right)= & \frac{\sinh \left(r x_{0}\right)}{\sinh (r x)}+ \\
& \frac{\pi_{\underline{x}, 0}\left(x_{p}\right)}{\sinh (r x)}\left[\sinh (r x)-\sinh \left(r\left(x-x_{0}\right)\right)-\sinh \left(r x_{0}\right)\right]
\end{aligned}
$$

Taking $x_{0}=x_{p}$ and using $\pi_{\underline{0}, x}\left(x_{0}\right)=1-\pi_{\underline{x}, 0}\left(x_{0}\right)$, one obtains

$$
\pi_{\underline{0}, x}\left(x_{p}\right)=\frac{\sinh \left(r\left(x-x_{p}\right)\right)}{\sinh \left(r\left(x-x_{p}\right)\right)+\sinh \left(r x_{p}\right)}
$$

which finally yields

$$
\sigma\left(x_{p}, x\right)=\frac{r \sinh \left(r x_{p}\right) \cosh \left(r\left(x-x_{p}\right)\right)}{\left(\sinh \left(r x_{p}\right)+\sinh \left(r\left(x_{p}-x\right)\right)\right)^{2}}
$$

The distribution of $S$ is then obtained from equation (28).

\section{E. An example of process with non connected span: the golden coupon problem}

So far, all the results that we have discussed apply to random walks with connected span. Here we consider a typical example of random walk with non connected span, which belongs to the family of intermittent random walks $[2,31]$. We assume that at each time step, the random walker jumps to a site drawn uniformly from the set of $N$ sites. For this process, determining the distribution of the territory explored before exit amounts to solving the following simple coupon problem. Assume that a coupon is drawn randomly out of $N$ different coupons labelled from 1 to $N$. The experiment is repeated until the coupon $N$ (the golden coupon) is drawn. Recall that the random walk defined above starts from a given site $s_{0} \neq 0$. Denoting $p(n)$ the probability that $n$ distinct coupons have been drawn before the golden one, one has:

$$
P(S=n+1)=p(n \mid n>0)=\frac{N}{N-1} p(n)
$$

This problem can be solved as follows. We denote by $S_{k, n}$ the number of surjections from $\llbracket 0, k-1 \rrbracket$ to $\llbracket 1, n \rrbracket$. The probability $p(n)$ of receiving $n$ distinct coupons before $N$ now reads:

$$
\begin{aligned}
p(n) & =\left(\begin{array}{c}
N-1 \\
n
\end{array}\right) \sum_{k=0}^{\infty} \frac{1}{N^{k}} S_{k, n} \frac{1}{N} \\
& =\left(\begin{array}{c}
N-1 \\
n
\end{array}\right) \frac{1}{N} \sum_{k=0}^{\infty} \frac{1}{N^{k}} \sum_{j=0}^{n}(-1)^{n-j}\left(\begin{array}{c}
n \\
j
\end{array}\right) j^{k} \\
& =\left(\begin{array}{c}
N-1 \\
n
\end{array}\right) \frac{1}{N}(-1)^{n} \underbrace{\sum_{j=0}^{n}(-1)^{j}\left(\begin{array}{c}
n \\
j
\end{array}\right) \frac{1}{1-\frac{j}{N}}}_{A}
\end{aligned}
$$

where $A$ can be shown to be given by

$$
\begin{aligned}
& A=(-1)^{n} \frac{n !}{(N-1) \ldots(N-n)} \\
& A=\frac{(-1)^{n}}{\left(\begin{array}{c}
N-1 \\
n
\end{array}\right)}
\end{aligned}
$$

yielding

$$
p(n)=\frac{1}{N}
$$

Finally, using equation (42) we obtain the strikingly simple exact result:

$$
\forall n \geq 2, P(S=n)=\frac{1}{N-1}
$$

Note that the result (45) can be interpreted as follows. Let us consider that the experiment is repeated until all different coupons are drawn at least once. This event eventually occurs with probability 1 . For symmetry reasons, the order of appearance of the golden coupon $N$ is uniformly distributed, which directly yields (45). 


\section{CONCLUSION}

To conclude, we have derived the full distribution of the territory $S$ explored by a 1-dimensional random walker before it exits a finite domain. This result applies to the general class of Markovian processes with connected span. We have demonstrated that this distribution can be expressed in terms of splitting probabilities only, which can in general be derived from Backward equations. We have applied our approach to various examples of random processes, which appeared in the literature in the context of target search problems. These include simple symmetric random walks, biased random walks, persistent random walks and resetting random walks. As a byproduct, we have provided explicit ex- pressions for the splitting probabilities of discrete persistent random walks and discrete resetting random walks, which were not known to the best of our knowledge. Il faudrait reciter ici je pense. We have finally discussed several extensions of our approach, namely to the case of reflecting boundary conditions, continuous processes, and an example of random process with non connected span. We now wish to inquire about higher dimensions and study $S$ thoroughly in different setups. Of note, our approach is by nature limited to 1-dimensional processes with connected span, for which the explored territory has a simple geometry parametrized by a single scalar parameter; the cases of higher dimensional problems, or of general processes with non connected spans call for alternative methods.
[1] B. Hughes, Random Walks and Random Environments (Oxford University Press, New York, 1995).

[2] O. Bénichou, C. Loverdo, M. Moreau, and R. Voituriez, Reviews of Modern Physics 83, 81 (2011).

[3] G. M. Viswanathan, E. P. Raposo, and M. G. E. da Luz, Physics of Life Reviews 5, 133 (2008).

[4] S. Redner, A Guide to First- Passage Processes (Cambridge University Press, Cambridge, England, 2001).

[5] R. Metzler, G. Oshanin, and S. Redner, First passage problems: recent advances (World Scientific, Singapore, 2014).

[6] A. J. Bray, S. N. Majumdar, and G. Schehr, Advances in Physics, Advances in Physics 62, 225 (2013).

[7] O. Bénichou and R. Voituriez, From first-passage times of random walks in confinement to geometry-controlled kinetics, Physics Reports 539, 225 (2014).

[8] S. Condamin, O. Bénichou, V. Tejedor, R. Voituriez, and J. Klafter, Nature 450, 77 (2007).

[9] A. F. Cheviakov, M. J. Ward, and R. Straube, Multiscale Modeling \& Simulation 8, 836 (2010).

[10] Z. Schuss, A. Singer, and D. Holcman, Proc Natl Acad Sci U S A 104, 16098 (2007).

[11] O. Bénichou, C. Chevalier, J. Klafter, B. Meyer, and R. Voituriez, Nat Chem 2, 472 (2010).

[12] T. Guérin, O. Bénichou, and R. Voituriez, Nat Chem 4, 568 (2012).

[13] T. Guérin, N. Levernier, O. Bénichou, and R. Voituriez, Nature 534, 356 (2016).

[14] M. J. A. M. Brummelhuis and H. J. Hilhorst, Physica A: Statistical Mechanics and its Applications 176, 387 (1991).

[15] M. J. A. M. Brummelhuis and H. J. Hilhorst, Physica A: Statistical Mechanics and its Applications 185, 35 (1992).

[16] M. Chupeau, O. Benichou, and R. Voituriez, Nat Phys 11, 844 (2015).
[17] O. Bénichou, M. Coppey, M. Moreau, P. H. Suet, and R. Voituriez, EPL (Europhysics Letters) 70, 42 (2005).

[18] G. H. Weiss and P. P. Calabrese, Physica A: Statistical and Theoretical Physics 234, 443 (1996).

[19] S. Condamin, V. Tejedor, and O. Benichou, Phys Rev E 76, 050102 (2007).

[20] S. Burov and E. Barkai, Physical Review Letters 98, 250601 (2007).

[21] S. Condamin, V. Tejedor, R. Voituriez, O. Benichou, and J. Klafter, Proceedings of the National Academy of Sciences 105, 5675 (2008).

[22] G. Weiss, Aspects and Applications of the Random Walk (Amsterdam, Netherlands: North-Holland, 1994).

[23] H. Larralde, P. Trunfio, S. Havlin, H. E. Stanley, and G. H. Weiss, Nature 355, 423 (1992).

[24] I. Dayan and S. Havlin, Journal of Physics A: Mathematical and General, 25, L549 (1992).

[25] M. R. Evans and S. N. Majumdar, Physical Review Letters 106, 160601 (2011).

[26] M. R. Evans, S. N. Majumdar, and G. Schehr, Journal of Physics A: Mathematical and Theoretical, 53, 193001 (2020).

[27] N. Van Kampen, Stochastic Processes in Physics and Chemistry, Third Edition (North-Holland personnal library, 1992).

[28] V. Tejedor, R. Voituriez, and O. Bénichou, Physical Review Letters 108, 088103 (2012).

[29] A. Chechkin and I. M. Sokolov, Physical Review Letters 121, 050601 (2018).

[30] A. Pal and V. V. Prasad, Physical Review E 99, 032123 (2019).

[31] O. Benichou, C. Loverdo, M. Moreau, and R. Voituriez, Physical Chemistry Chemical Physics 10, 7059 (2008). 